\title{
The Use of Observation and Interview Methods for Assessing Issues in Patient Care in the Resuscitation Unit of a Level-1 Trauma Center
}

\author{
Joseph Sharita, Lorgia McCane ${ }^{\mathrm{b}}$, Carl I. Schulman ${ }^{\mathrm{c}}$, \\ Jill M. Graygo ${ }^{d}$ and Jeffrey S. Augenstein ${ }^{e}$ \\ ${ }^{a}$ University of Miami, Department of Industrial Engineering, P.O. Box 248294, \\ Coral Gables, FL 33124-0623,305-284-6472, jsharit@miami.edu \\ ${ }^{b}$ University of Miami, Leonard M. Miller School of Medicine, \\ lmccane@trauma.med.miami.edu \\ ${ }^{c}$ University of Miami, Leonard M. Miller School of Medicine, \\ DeWitt Daughtry Family Department of Surgery, cschulman@med.miami.edu \\ ${ }^{d}$ University of Miami, Leonard M. Miller School of Medicine, \\ jgraygo@trauma.med.miami.edu \\ ${ }^{e}$ University of Miami, Leonard M. Miller School of Medicine, \\ DeWitt Daughtry Family Department of Surgery, jaugenst@trauma.med.miami.edu
}

\begin{abstract}
Although traumatic injury is the leading cause of death in the U.S. for people between the ages of one and 44, we lack important knowledge about how the various activities and processes within the resuscitation units of trauma care systems can impact the management of patient care. This article reports on a research study that involved the complementation of observation and interview methods for identifying and assessing a broad array of issues and concerns within this highly complex critical care setting in a large level-1 trauma center. Data from observations were collected on 27 days, and subsequently used to guide semi-structured interviews with 22 health care workers representing different specialties responsible for patient care within the resuscitation unit. The complementation of observation and interview data afforded the opportunity to validate issues that were observed while providing a richer understanding of these issues as a basis for formulating intervention strategies.
\end{abstract}

Keywords: resuscitation unit, quality of trauma care, observation study, interview study, integrating qualitative methods

\section{INTRODUCTION}

Traumatic injury is the leading cause of death in the U.S. for people between the ages of one and 44, and its resulting loss of productive life-years exceeds that of any other disease with societal costs of $\$ 406$ billion dollars annually. ${ }^{1}$ However, despite the importance of trauma care and the large and increasing outlay of monetary resources for 
treatment at trauma centers, ${ }^{2}$ we lack important knowledge about how the various activities and processes within these systems can impact upon the delivery of care for patients with traumatic injuries. This is especially true for the resuscitation units of large, level-1 trauma care systems. In these highly fluid high-hazard environments interactions among health care workers from widely different areas of specialization and various hospital units can occur under rapidly changing and unpredictable conditions, ${ }^{3,4}$ providing the pathways by which normal human and system variability can translate into poor outcomes. ${ }^{5-7}$

This article reports on the complimentary use of observation and interview methods for identifying and assessing potential concerns within the resuscitation environment that could adversely impact patient care. The objective of the observation component of the study was to identify issues related to patient management at both the "sharper" patient-provider and the broader system levels, as well as to capture relevant contextual details associated with these issues. This information was then used to guide semistructured interviews with a broad class of medical providers responsible for patient care in the resuscitation unit in order to validate and gain a richer understanding of potential concerns.

While field observations represent a very fundamental qualitative research method for which well established principles and guidelines applicable to a wide variety of problem domains,${ }^{8-9}$ including health care, ${ }^{10}$ have evolved, their use in health care settings, while prolific and very valuable, has generally been directed toward highly specific problems. For example, observational methods have been used to investigate specific issues such as patient hand-offs, ${ }^{11}$ the frequency of drug administration errors, ${ }^{12}$ problems with equipment interaction, ${ }^{13}$ operating room and emergency room communication patterns, ${ }^{14,15}$ and nurse interaction with bar code medication administration technology. ${ }^{16}$ In many of these kinds of studies, observations are usually directed at revealing work contexts relevant to the specific questions of interest; seldom are such methods used to understand less structured and more uncertain situations.

As noted by Carthey, ${ }^{17}$ large critical care environments such as intensive care units (ICUs) present unique challenges for performing structured observational research. In evaluating patient care management in the highly complex and dynamic resuscitation environment of a large level-1 trauma center, these challenges become even more magnified. These environments require, among other considerations, (a) the coordination of health care providers representing a very broad array of specialty areas, (b) the ability to adapt rapidly to highly variable patient loads and to patients who often arrive with less than complete information regarding their medical histories and current medical conditions, (c) the ability to track and communicate information about impending patients arriving through a variety of modes of transport, and (d) coordination with specialists and support services provided by other hospital units. To identify potential concerns for patient care within this setting, observational methods need to be tailored to consider a much broader array of work contexts and issues than those that are typically targeted in most health care studies.

Further complicating the prospects for obtaining observational data in these resuscitation environments are a host of logistical issues. These include potential concerns by staff over job security and litigation, and thus the possibility that they do 
not agree to being observed-concerns that can hinder research efforts. ${ }^{18}$ In addition, the Joint Commission's policies on videotaping ${ }^{19}$ as well as concerns by the University's Institutional Review Board (IRB) for patient and staff confidentiality will generally preclude filming or the use of audio-recording devices by outside observers.

In the following sections, a detailed description of the methodology used for collecting and analyzing observational data within the resuscitation environment is presented, followed by a summary of the results of the observations. A sample of the data derived from subsequent structured interviews, which was guided by several emergent themes from the observation study, is then presented. The overall study goal was to gain insight into directions for improvement of patient care in this setting by identifying and examining an array of potential concerns capable of undermining health care system performance. ${ }^{20}$

\section{METHODS}

\section{Study Site}

The study was performed in the resuscitation unit of the Ryder Trauma Center (RTC), a four-story, free standing level-1 trauma center associated with the University of Miami Miller School of Medicine and Jackson Memorial Hospital. This unit contains six resuscitation rooms and six observation/holding beds, and its own dedicated administrative and nursing station. The RTC has six operating rooms, its own 20-bed surgical intensive care unit, burn unit, and rehabilitation unit, and provides the largest trauma/critical care fellowship in the United States. Jackson Memorial Hospital, to which the RTC is linked, is the largest hospital in the country, and frequently runs at maximal occupancy and understaffed.

\section{Consenting, Protecting, and Informing Medical Providers in the Resuscitation Unit}

Recognizing that the resuscitation unit (RU) is a very fluid environment in which many providers flow in and out depending on the nature and extent of patients' injuries, obtaining signed informed consents for all staff members who might be observed during any particular observation period was unrealistic. Thus, a waiver of informed consent for the study participants was required by the University's IRB, who approved this study. In addition, to protect identifiable research information from forced disclosure, we were granted a Certificate of Confidentiality from the National Institutes of Health (NIH). This allowed participants to refuse to disclose information that could adversely impact their employability, insurability, or reputation and thus promoted participation in the study.

The waiver of informed consent, however, necessitated demonstrating that participants were adequately informed about the study as the appearance of study personnel in the RU without the knowledge and support of the staff could have damaging implications for the collection of data. A number of processes were used to inform the providers about the study, including postings and e-mail distribution of a memo summarizing the study and its objectives; presentations of the study to RU staff during shift changes; and, once observations began, informal presentations of the study in the RU on a one-to-one basis to any medical provider that requested it. 


\section{Training Observers and Collecting Observational Data \\ Observers}

Four observers took part in the study: two were clinicians and two were human factors professionals specializing in health care systems. One clinician and one human factors specialist were usually assigned as observers during any given observational period.

\section{Sampling Strategy}

The sampling strategy that was adopted was to allocate responsibility between the observers such that one observer was to focus more directly on patient care activities while the other focused on system-related activities. For example, the observer focusing on patient care in a resuscitation room may note (a) the time spent looking for needed supplies, (b) issues with patients being positioned or stabilized, (c) the potential disrupting effects of overcrowding in the room on communication among providers, (d) workplace design factors that could compromise the ability to obtain x-rays from the patient, and (e) the nature of the leadership and its impact on communication.

The observer allocated to system issues may (a) attend to problems with transmissions regarding incoming patients or to communication between $\mathrm{x}$-ray technicians in radiological services and nurses in the RU, (b) shadow nurses who are trying to contact specialists from other hospital units, and (c) shadow providers as patients are transported to the heliport (on the roof of the trauma center) to note the nature of communication with emergency transport workers. However, observers were aware that these roles often overlapped and needed to be adjusted depending on other factors such as the busyness of the unit or the severity of patient injuries.

A debriefing followed each period of observation wherein the observers compared notes and clarified unclear issues. It also provided them with the opportunity to discuss and reassess strategies concerning the coordination of observations in the face of changing RU conditions.

\section{Materials}

All data were collected on handwritten notepads as the use of audio or video recorders in this environment was not permitted. Although electronic devices exist for possibly effecting more rapid recording of data, such devices, like those used to collect video or audio information, call attention to staff and potentially arouse suspicion. The use of eight-by-five inch notepads enabled observers to more easily keep these materials out of view when notes were not being taken. They also allowed for data collection "onthe-run," which typically occurred when moving from one area to another within the trauma care system.

\section{Familiarization with the Resuscitation Unit Environment}

Prior to the formal collection of data the observers became familiarized with the overall organizational structure of the resuscitation unit, including all units to which it could interface to. In addition, the various forms used by medical providers for patient documentation were reviewed. Because it was also essential that the observers be capable of discriminating between the various provider roles (e.g., nurse, second-year resident, senior resident, fellow, attending surgeon, consultant, anesthesiologist, social 
worker, emergency special care associate, x-ray technician, emergency transport worker), the initial meetings were also directed at establishing the different activities generally performed by these providers.

Five preliminary observations were performed prior to the formal collection of data to enable the observers to become better acquainted with the dynamics of activities within the resuscitation unit. These observations also helped the observers determine how to best situate themselves within this setting for note taking, and how to recognize appropriate opportunities for querying a provider in order to clarify events or procedures.

\section{Development and Use of an Observation Guide}

Given the complexity of the resuscitation environment and the objectives of identifying a broad array of issues related to the quality of patient management, it was necessary to provide the observers with an organizational framework regarding what constituted relevant data for collection. Toward this end, an "Observation Guide" was developed. Prior to the development of this tool, a listing of 52 issues related to trauma care was compiled and reviewed by the study's investigators based on the existing literature, past experiences by the study investigators in performing observations in critical care settings, and documented human factors and ergonomic considerations in hospital environments. This listing of issues formed the basis for the types of observational data contained in the guide. The guide was organized into five sections. Table 1 presents a few examples of some concerns addressed within the first three sections of this guide.

Development of the guide was an iterative process. A preliminary version of this guide was reviewed by the Department of Risk Management at Jackson Memorial Hospital who helped identify concerns that we omitted and, given their sensitivity to legal issues, also helped ensure that the directives to observers as stated in the guide reflected, as much as possible, objective rather than subjective appraisals.

Table 1. Example of items from three of the main sections of the Observation Guide

Section 1. Observations associated with the impending arrival and actual arrival of a patient into the Resuscitation Unit

- How is an impending patient arrival "broadcast?" Is this broadcast adequate to apprise all required providers? Does anyone state that they did not hear the broadcast? Does anyone state that they were unaware that a patient was being brought in? What mechanisms exist to confirm that the "broadcast" is received and acted upon?

- Do any providers have to inquire further from the transport team bringing the patient into the Resuscitation Unit (e.g., paramedics) to obtain additional information? Are these inquiries routine? Is that information reported in any other way (i.e., in writing)? Is verbal information written into the patient's chart? Is there further follow-up and clarification required or is the information provided sufficient to allow the providers to proceed? How long does the transport team stay after hand-off? What activities, paperwork, or other actions do they take before they leave the area? Are they asked to assist in patient care after hand-off?

contunued over 
$\overline{\text { Section 2. Observations associated with patient care within the Resuscitation Unit or the adjacent }}$ patient observation area

- What is the style of team communication during patient care in the trauma bay room?

a. A provider (e.g., senior resident, fellow, or attending) is dictating care and there is limited or no questioning by other team members.

b. A provider is dictating care and there are interactive responses or inquiries (e.g., of commands or plans) by other team members.

c. The atmosphere is quiet—everyone is going about their functions more or less independently.

- Are requested supplies or equipment readily available for patient care? Are there any supplies or equipment that must be obtained from outside the unit? If so, what are they and from where are they brought in? How long does it take to receive them once a request is made?

- Are there any imaging studies ordered while the patient is in the Resus or the observation area? Are any imaging studies done at the bedside (i.e. portable X-ray) or in the unit? If the patient is required to leave the unit, how is the transport accomplished? How much time does it take for a transport team to arrive? What provider, if any, accompanies the patient? How long does it take for the patient to be returned to the unit? How long does it take to receive the imaging studies? In what form are they delivered? Is an initial reading provided along with the image? How is that reading reported and documented? Are there any requests made by the ordering provider or other responsible provider to determine the status of an imaging study request? What actions, if any, are taken in response to such an inquiry?

- Are there any events during the provision of patient care in the unit that either distract or interrupt the team delivering care? What is the nature of that event? How long is the interruption? Is the team able to promptly re-focus on patient care?

- Are there any observed, observable, or expressed indications regarding equipment design, accessibility and usability of equipment, workplace design, process design, or process flow that should be identified and further evaluated? Are there any occupational safety hazards, observed or expressed, that should be identified and further evaluated?

Section 3. Observations associated with documentation activities within the Resuscitation Unit

- What are the methods used for documentation (e.g., handwritten personal notes, writing onto the patient's chart, use of a computer-based device, filling out a standardized form that will be inserted into the patient's chart)?

- Are there any statements made regarding technical difficulties with any equipment used for documentation activities?

- Are there any events that interrupt documentation activities? If yes, how long was the interruption and did the provider complete the documentation? 
The Observation Guide served as the primary training platform for the observers. As part of their training, observers were given a variety of example scenarios that reflected the various concerns listed in the guide. The preliminary observations served to further reinforce the material in this guide. The Observation Guide, however, was not intended to be used as a checklist during actual observations. We believed that the unpredictability, rapidity, and scope of activities in the resuscitation environment required a much more flexible approach to data collection, and that the guide could best serve this objective by mentally preparing the observer for note-taking. Thus, it was understood that the guide was not expected to contain an exhaustive list of issues, and any additional issues that were identified during the course of the observations were documented if they were considered to be consistent with the study's goals.

\section{Level of Detail in Observations}

Nemeth, Cook, and Woods ${ }^{18}$ have called attention to the importance of studying how health care workers adapt in unique ways to the demands and constraints of the work environment, often by resorting to artifacts that help them organize, plan, or tailor various resources to meet situational demands. Although they note that most details in the study of cognitive work are not significant, they also emphasize that opportunities for improving patient care rely on understanding how such adaptations can both accommodate and influence the changing cognitive demands health care workers face.

This consideration raises an important methodological issue, namely, specification of the kinds of observations that constitute an appropriate level of detail. While the Observation Guide was designed in large part to resolve this issue, for several reasons it was not designed to explicitly capture the kinds of details that might underlie an individual provider's adaptive strategies. First, the primary goal of this study entailed, to some extent, trading-off a narrow focus on idiosyncratic adaptive behaviors and their associated artifacts with a methodology that emphasizes identifying broader concerns. Second, the resuscitation setting was not particularly conducive to identifying such behaviors, especially when at most only a small subset of individuals being observed might be present during subsequent observation periods. Finally, we hoped to capture some aspects of adaptive coping strategies from interviews that were guided by conditions and issues observed in the field study, although we recognized that such data may not be as revealing as observations of actual work contexts. ${ }^{21}$ Overall, these considerations point to the importance in this study of establishing as a goal the design of an observation protocol that is neither too narrowly focused nor too generic.

\section{Observation Periods}

A total of 27 observations within the resuscitation unit on 27 different days were carried out. These observations totaled about 80 hours. One-third of the observations took place during the weekend: two were in the morning, one was in the afternoon, and six were in the late evening. The remainder of the observations took place on weekdays at various times of the day. 


\section{Analysis of Observational Data}

Extraction of Data from Observations

A stepwise mapping process, which is summarized in Table 2, was developed for extracting and compiling data from the observations. The process began by capturing the details of patient care activities in handwritten notes, which always included the time at which activities took place. The notes for each observation were then reviewed and transcribed to a more permanent computer record, which was examined for what the investigators considered "notable scenarios." These scenarios were identified based on the set of issues that were used to develop the Observation Guide or any additional noteworthy issues that were observed.

Table 2. The stepwise mapping process of identifying and assigning issues to observations of patient care activities

1. Each observation $\rightarrow$ time-coded handwritten "rough" notes

2. Rough notes $\rightarrow$ transcribed written notes

3. Transcribed notes $\rightarrow$ highlighting of notable scenarios

4. Notable scenarios $\rightarrow$ extraction of key elements

5. Key elements $\rightarrow$ creation of summary tables

6. Summary tables $\rightarrow$ assignment of numerical codes

7. Numerical codes $\rightarrow$ frequency counts of issues

Table 3. Part of a summary table for a particular observational period showing the key elements that were extracted and the coded issues that were assigned to these key elements ${ }^{1}$

\begin{tabular}{|c|c|}
\hline Codes & Key Elements \\
\hline 3 & $\begin{array}{l}\text { ANRs and } \mathrm{N} \text { were not communicating their tasks and intentions to one another, } \\
\text { creating uncertainty and task delay. ANRs did not know } \mathrm{N} \text { was done and } \mathrm{N} \text { did not } \\
\text { know they were waiting for her. }\end{array}$ \\
\hline $17,6,20$ & $\begin{array}{l}\mathrm{N} \text { comments to lab tech that moving patient to place XR film underneath patient } \\
\text { was inappropriate in case patient had pelvic fracture. No response from lab tech. }\end{array}$ \\
\hline 3,6 & $\begin{array}{l}\mathrm{CT} \text { tells } \mathrm{N} \text { in } \mathrm{RU} \text { that } \mathrm{CT} \text { is ready. When } \mathrm{R} 2 \text { inquires several minutes later as to its } \\
\text { availability, } \mathrm{N} \text { says it isn't. }\end{array}$ \\
\hline 15,16 & $\begin{array}{l}\mathrm{R} 2 \text { determines that anesthesia must return to this case. Also, RT left unit and is } \\
\text { needed and } \mathrm{R} 2 \text { is frustrated by this occurrence. }\end{array}$ \\
\hline $6,15,20,16$ & $\begin{array}{l}\text { CT arrives to pick patient up but patient isn't ready. CT pushed away a respiratory } \\
\text { embolism case to do this case and was frustrated. } \mathrm{N} \text { blames delay on waiting for } \\
\text { RT. CT left without the patient. }\end{array}$ \\
\hline 3,8 & $\begin{array}{l}\text { When RT arrives, R } 2 \text { argues with her saying it was not a good idea to leave before } \\
\text { a chest XR comes back. RT says she didn't hear the pager despite standing next to } \\
\text { it and being paged } 4 \text { times. }\end{array}$ \\
\hline
\end{tabular}

$\mathrm{N}=$ nurse. $\mathrm{ANR}=$ anesthesia resident. $\mathrm{RT}=$ respiratory technician. $\mathrm{PM}=$ paramedic. $\mathrm{R} 2=$ second-year surgery resident. $\mathrm{CT}=\mathrm{CAT}$ scan. $\mathrm{XR}=\mathrm{x}$-ray. $\mathrm{RTC}=$ Ryder Trauma Center. $\mathrm{ER}=$ emergency room.

${ }^{1}$ Explanations of some of these codes are given in the text. 
Next, "key elements" were extracted from the notable scenario descriptions. These key elements were then compiled into a "summary table." Thus each observational period had associated with it a summary table containing a sequential listing of key elements. A part of a summary table associated with one of the observational periods is shown in Table 3. A description of the larger contexts associated with this sequence of key elements, as derived from the notable scenarios contained within the transcribed handwritten notes, is presented in Table 4.

\section{Table 4. A fuller description of the contextual details ${ }^{1}$ corresponding to the key elements in Table 3}

A patient (P1) arrives by helicopter. The paramedic provides no information to the medical providers during the transfer of the patient [possibly because he was given none by the people who rescued the patient, which is not uncommon in transport by helicopters] and immediately leaves. In the elevator, during transport from the helipad to the RU, the R2 asks the patient how the accident happened. P1 answers that he tripped over a cable when operating welding equipment and fell off a second-floor balcony. When the TA arrives N1 gives him the report and says "Paramedic judgment;" the TA rolls his eyes. Both providers realize that this GCS 15 patient was over-triaged. [This type of decision reflects the conservativeness that is often displayed when dealing with such emergencies; the slightest uncertainty often translates into decisions by rescue workers to bring the patient to the RU.]

As a team (R2, N1, PR, SW, RT, N2, X-Ray tech) attends to P1, N1 continues the cynical posture concerning over-triage: "Not even a scratch? Did he hit his head at all?" The R2 performs an ultrasound, N2 begins an IV and orders lab tests, the PR queries P1 for information, and the SW fills out forms. X-rays were taken of the chest and pelvis [these are the most routine $\mathrm{x}$-rays taken of patients brought to the RU]. N1 draws femoral blood and prepares tubes for various blood tests. $\mathrm{R} 2$ indicates to $\mathrm{N} 2$ that a $\mathrm{C}$-spine is needed because $\mathrm{P} 1$ complained of pain. The ESCA then enters the bay room to inform the team that the TA did not want blood. The R2 responds "He did not tell us not to do a blood gas," to which N2 adds "He needs to tell us." The ESCA exits to call the TA and determine the TA's intentions, and returns informing R2 and N2 that the order was not to do blood and that the TA thought they heard him.

At this time, a radio transmission is broadcast concerning a female (P2) in a MVC, GSC 12, giving an ETA of "Now." This upsets N3 because there was no prior communication that the arrival would occur at that particular time. [Depending on the case, preparation for patients is essential in the RU, especially for nurses and anesthesiologists.] R2 and N3 go to the helipad. During transport to the RU in the elevator R2 asks the PM: "Is she breathing?" The PM responds, "Yes, this was a MVC, they found her in the back seat," and then adds "Possible head injury." R2 checks P2's abdomen in the elevator, and as they enter the RU R2 asks angrily, "Where is anesthesia?" N2 responds, "They should be here by now." [Because airway, breathing, and circulation - the ABCs - are of primary concern in many trauma cases, the presence of anesthesia is critical.] A team consisting of the TA, R2, N3, x-ray tech, and the SW are attending to P2. N3 indicates that "Rescue gave her a sedative because she was combative." Anesthesia arrives and R2 briefly presents the case to the (three) ANRs. N3 places an IV in P2, during which time the ESCA twice asks if the rescue report was received, to which N3 responds "No." Three ANRs are involved intubating P2: one holds P2's head, one holds the tube, another passes the instruments; one ANR also cleans the wound. [It is not uncommon to have 3 or even 4 anesthesiologists in the room as they feel communication concerning needed equipment is best carried out among 
themselves rather than through nurses.] A few minutes later the R2 is on the phone with a medical provider who is requesting a consult on the hospital floor. [This type of interruption is very common as the R2 is often called to serve as a consultant on various cases throughout the hospital.] While still on the phone, R2 speaks to F1 about the consult request and gives F1 details about P2, to which F1 responds "She will not survive an operation. Go examine her and then order a CT."

Following intubation of P2, the ANRs wait for N3 to finish her tasks (IVs, etc.) before proceeding with their other tasks. Finally, an ANR asks N3, "Are you done?" N3 replies, "I have been done, she's ready." [The lack of communication between anesthesiologists and nurses is not uncommon.] During this time, N2 wants an X-ray for P1 performed before an X-ray for P2, even though P2 is a priority. It seemed as if $\mathrm{N} 2$ wanted to get done with her work. Three minutes later, the $x$-ray tech arrives and moves P2 to insert a film underneath her; N3, watching this process, informs the technician: "It is not a good idea to do that in case she has a pelvic fracture." The technician does not respond. [Friction has been reported by Radiology between radiology technicians and RU nurses: "There is always that underlying conflict between the nurses and technologists where the nurses feel that they are more important than the technologists."]

Nine minutes later, $\mathrm{N} 1$ calls in a CT scan to Radiology for P2 and confirms that CT is ready. R2 auscultates $\mathrm{P} 2$, who is combative; $\mathrm{N} 3$ administers more sedative to P2; blood gas results come back. When R2 asks if CT is ready N1 now says "No," and CT is called again, this time by R2. RT and N3 disagree over saturation rates: "We don't necessarily have to have SAT 100," says RT. N1 calls out "I need a quick brain on it;" F1 comes in and says "She needs to go down." R2 says anesthesia needs to return. X-ray results are reviewed by R2 and F1; pelvis and chest are negative. F1 tells R2: "Do CT scan of the head and then full back." He then looks back at the Xrays and says, "The problem is in her head." N3 asks R2 to page RT. N1 obtains a portable monitor and places it on a stretcher (in preparation for transporting P2 to CT, which requires accompaniment by an RT). R2 makes a call and says, "It's amazing that it takes 10 minutes for the helicopter to get her and respiratory is not here." When CT arrives to pick P2 up he asks "Are you ready?" When he realizes they are not he says "I pushed a respiratory embolism case to do her." N3 responds "We are ready. We are just waiting for respiratory." I thought you were ready to roll," the CT operator says. CT leaves without P2. [This reflects the poor communication between resuscitation and CT. The CT that is in the trauma center is a scarce resource that is also dedicated to the ER, and if Radiology presumes a patient is ready, they forego other patients that may need the CT.] N3 says to R2: "This is ridiculous," referring to the RT's absence. Five minutes later the RT arrives. R2 argues with the RT: "It's not a good idea to leave before a chest x-ray comes back. We paged you four times." RT responds, "I did not hear the page. I was standing next to the pager." R2 and RT get into an argument over RT's absence. N3 intervenes saying "Come on, let's go." P2 is taken to CT. About 20 minutes later the R2 returns from the CT.

Forty minutes later there is a radio transmission, ETA 3 minutes, of an older female (P3) pedestrian hit by a van, GCS 3 (very serious). Shortly thereafter, P2 is brought to the OR for brain surgery (it had been determined that she was pregnant, which had important implications for her care).

\footnotetext{
${ }^{1}$ Many medical details from the actual transcription are intentionally not included. Bracketed passages reflect additional commentary.

$\mathrm{RU}=$ Resuscitation Unit. ETA = estimated time of arrival. GCS = Glascow Coma Scale. MVC = motor vehicle collision. CT = CAT Scan. R2 = second-year surgery resident, who is in charge of the RU unless a more senior physician is present. $\mathrm{P} \#=$ patient number. $\mathrm{N \#}=$ nurse number. $\mathrm{TA}=$ attending trauma surgeon. ANR = anesthesia resident. $\mathrm{SW}=$ social worker. $\mathrm{F} \#=$ fellow number. ESCA = emergency special care assistant. $\mathrm{PM}=$ paramedic.
} 


\section{Coding Key Elements and Establishing Consensus}

The key element data extracted from the observations were independently coded by two of the study team members who were most familiar with human factors and systems issues and who had prior experience in collecting observational data in critical care environments. The coding process entailed assigning to each key element in each observation summary table numbers corresponding to one or more of the 52 issues previously identified and used as a basis for development of the Observation Guide. Multiple codes, if relevant, were deemed necessary as activities or situations with implications for patient care in this environment could be the result of a complex interaction of factors.

The 27 observation summary tables yielded 233 key elements to which codes were to be assigned. In 189 of these cases (about $81 \%$ ), there was agreement between the evaluators in at least one of the assigned codes. Fourteen of the 52 pre-identified issues were never identified as relevant by either evaluator and thus never used to code any key elements. In studies such as these where coders are faced with the task of assigning, from a relatively large collection of issues, multiple codes to any given occurrence, the use of Cohen's Kappa is problematic and adopting percent agreement (on at least one code) is a reasonable approach to estimating observer reliability. ${ }^{22}$

Wherever discrepancies existed between the coders, consensus was arrived at through discussion. To facilitate these discussions, the more complete texts (typically, the "notable scenarios") from which the key elements were extracted were referenced. In some cases, issues were redefined (i.e., relabeled) or aggregated, or new issues were generated. For those key elements in which further clarification was required due to considerations that were highly clinical in nature, consensus was arrived at using a third evaluator who was one of the clinical observers. Ultimately, a total of 29 issues were assigned to the key elements (Table 5).

Table 5. Individual counts in descending order of each of the 29 issues extracted from all observation summary tables

General frustration (53)

Organizational/administrative issues (36)

Team communication (27)

Patient safety (26)

Diagnostic services (24)

Preparation for a patient (24)

Interdepartmental communication (22)

Skills requirements (21)

Too few/many providers in bay room (19)

General/worker safety issues (16)

Organizational climate (15)

EMS-Resuscitation communication (14)

General confusion/distraction (14)

Resuscitation equipment readiness (13)

Prioritization of patient care (11)
Team structure (9)

Distraction due to patient combativeness (9)

Workload (8)

Training issues (8)

Workplace design issues (7)

Documentation activities (7)

Consult delay (6)

Diagnosis issues (6)

Supplies availability (5)

Intimidation (5)

Pain management (4)

Medication issues (2)

Special needs (2)

Fatigue (2) 


\section{Complementation of Observation and Interview Methods}

The purpose of administering follow-up interviews to trauma care providers who work in the RU was to obtain a more comprehensive appraisal of the issues, and contextual details associated with those issues, that had been exposed in the observation study. This complementation of qualitative methods also afforded a basis for validating the issues that emerged during observations in this unit. ${ }^{4}$

Twenty-two health care workers from the RU representing eight different subcategories of workers participated in the interview component of the study. These included nine physicians specializing in trauma care (four attending trauma surgeons, two fellows, and three residents), three anesthesiologists (two attending physicians and one resident), four nurses, three emergency special care associates, and three workers from radiological services (x-ray/CT technicians). Each participant was interviewed individually at a time and in a private location selected by the participant, and provided a signed informed consent allowing for audio-taping.

The interviews were semi-structured. This enabled the interviewers, who consisted of two of the observers, the freedom to interject and ask additional questions (the nature of which was often motivated by their observations in resuscitation) in response to the answers provided by these workers. Relatively distinctive sets of questions were constructed for each of the eight health care worker sub-categories (some overlap naturally occurred between certain sub-categories, for example, between trauma attending surgeons and trauma fellows), whose content was guided by issues and concerns that emerged from the observational data.

\section{RESULTS AND DISCUSSION}

\section{Summary of Key Elements from Observations}

As shown in Table 3, key elements in some cases were assigned either one or two codes (corresponding to one or two issues), and in other cases multiple codes were assigned. For example, the numerical code of 3 corresponded to "team communication," 6 corresponded to "diagnostic services," 16 corresponded to "interdepartmental communication," 17 corresponded to "skills requirements," and 20 corresponded to "patient safety issues."

The 29 issues that were ultimately assigned were counted, as indicated in step 7 of Table 2. The individual counts of each of these issues, in descending order of occurrence, are given in Table 5. The issue of "frustration" was noted by observers only if there were overt demonstrations, by actions or speech, of such feelings. With respect to some of the other issues with relatively high frequencies of occurrence, an organizational/administrative issue was assigned when the observed issue was believed to be due to the way the trauma care system was organized or to administrative policies that potentially had adverse implications for care; team communication referred to any type of communication among RU staff that could possibly have a negative implication for patient care; and diagnostic services and patient safety issues were often identified in conjunction with other issues such as skills requirements and organizational or administrative issues. 


\section{Some Further Analysis of Emergent Themes from Observations}

In this section, several "emergent themes" from the observation study are examined further based on a sample of the responses derived from the interviews. One such theme related to the "readiness" of the unit with respect to the issue of preparation for a patient. The anticipation of needed care, which is critical to the effectiveness of medical providers in resuscitation, entails reliable broadcasting and receiving equipment to inform staff of impending arrivals, the communication of unambiguous and relevant information from rescue personnel over the various broadcast media, ensuring that key providers are aware of broadcasts, appropriate inquisitiveness on the part of the RU's leader in response to broadcasts, and the readiness of equipment.

Observations had indicated the need for improvements in each of these areas. Very little communication between emergency transport (e.g., paramedics) and the residents and fellows who received patient hand-offs from these personnel was observed, and medical providers had different perspectives on this issue. One anesthesiologist felt the communication of field information was "very, very, important," and noted "I think the EMS system actually does a pretty good job in giving out as much information as possible to us" with the most critical information desired consisting of "mechanism of injury, if they are really critical, did they lose a pulse on the way over here, and did they find a patient without a pulse and do CPR on them." Attending trauma surgeons, however, did not generally attribute much value to communication by rescue personnel. As one attending stated: "They (rescue workers) come to us trying to give a report. In all honesty I generally find their information worthless. I would rather they get out of there as quickly as possible. I know that we are supposed to have this great sign-off but they all have different levels of ability and I would rather have someone tell me just the briefest of information." Overall, the data indicated that guidance in communication on both ends - in the questions asked by physicians, even as the patient is being rolled in, and by information offered by emergency transport workers-appears warranted.

With regard to equipment readiness, one nurse indicated: "Anesthesia, before the trauma, they should come in. Usually the techs set up the suction canisters but I think they are just putting them there, they are not setting them up properly, so they are

hooking them wrong so the suction is not working, they haven't closed all the caps, so they are not working. Anesthesia should come in, if they know they are going to intubate, to make sure they have suction, get their meds, etc." As evident below, a lack of communication between nurses and anesthesiologists potentially undermined teamwork in this setting with blame extending in both directions.

The readiness of the unit was also observed to be impacted by diagnostic (radiology) services (as implied in the expanded scenario descriptions presented in Table 4), especially in light of the fact that some trauma surgeons, given the current climate of litigation, are demanding extremely high assurances of their diagnoses and requesting "man scans"-CT scans of the entire body. The data indicated that long waits by multiple patients for CT machines was not uncommon, and stemmed from both the need for more equipment and greater reliability in this equipment (these devices were often not functioning). This suggested the need for cost-benefit analyses that can more clearly indicate the benefits to patient care of increased (beyond monthly) preventive maintenance (for these 24/7 machines that are highly sensitive and must endure 
enormous abuse), and greater equipment availability for coping with high volume or multiple arrivals with critical injuries, especially for cases where it would be illadvised, due to patient safety concerns, to transport patients to CT located in the main hospital wing.

The issues surrounding teamwork and communication in health care systems have received considerable attention in recent years and many challenges, especially in education and assessment, still lay ahead. ${ }^{23}$ Our observations suggested that this is probably even more the case in the resuscitation setting, where the nature of health care is somewhat more fragmented and can reflect many different disciplines and departments. Teams can dynamically vary in size from two or three medical providers to over 20, depending on the situation. This essentially results in more loosely coupled teams made up of clusters of sub-teams. Patient care in this environment was also observed to be conducive to team or sub-team members becoming compartmentalized (overly focused on their activities). To enhance more global awareness among individuals or sub-teams, some degree of "broadcasting" of critical actions or decisions may need to become part of the process of patient care to ensure that a "common ground" is established. ${ }^{14}$

Related to the issue of communication was the rotational system that was in place. In this system, new residents are brought in every month but are often unfamiliar with or lacking in skills in trauma care procedures. While the presence of an attending trauma surgeon typically induces a greater sense of overall team leadership, these providers are not always present, and it was also not uncommon for more senior residents to be called away for consults throughout the hospital or to the operating room. Consequently, there were times when these second-year residents needed to assume the position of team leader in the RU. Fortunately, as indicated by observations and validated in interviews, redundancy in the form of highly skilled nurses and more experienced trauma physicians lessened this risk to patient care. Improved training could help ensure that the system is not vulnerable as new residents are rotated into unfamiliar trauma care environments, possibly using online modules and other media to provide needed knowledge.

The frustration of having a second-year "rotating" resident as a leader in a resuscitation room was voiced by one of the attendings as follows: "You're talking about the second year (resident)? Well, that's a problem because...their diagnostic and problem-solving skills are highly variable, and some are zero, none. I think that having a second year in that role is a mistake; we were forced to do that for staffing issues, and a second year is not mature enough to be in that role...these people are too junior to be managing these teams...they're pushed around...they're either emergency medicine residents and don't really grasp what we're thinking as surgeons or they're residents (from a different hospital), and they're a caliber below... and the second year, even our own residents, it's the rare resident that can really manage that place." On this same issue, another trauma attending physician noted, "If we are all in the operating room and (the rotating residents) are in resuscitation when the first trauma patients come in, how do they do an ultrasound, how do they know what the priorities are, what the options are? I think it is very wrong, but the problem is that they are taking away our surgical residents to go to other services. In a way, we are paying for our success." 
Nurses were also acutely aware of this team leadership issue, and were often the ones observed to be instructing and guiding these resident physicians. One trauma nurse recommended that "It might be better for them to come for longer times, for six weeks or two months at a time, instead of one month. That gives them time to get acclimated and actually to be functional for a while before we send them away and we get the next person."

Issues with team leadership, as well as with communication, were also evident from the perspective of the anesthesiologists. As one anesthesiologist remarked, "The ancillary staff gives priority to the surgeons, even if at the moment the most pressing issue is the airway. A lot of times they just turn their backs and leave anesthesia on their own; we're asking for stuff and they're not necessarily responding...the main problem is a lack of understanding of others' priorities; I guess the only way to (fix) that is training." This physician went on to say, "Because this is a teaching hospital, sometimes communicating with the nurses and residents can be a major issue...particularly the second-year rotating residents, and the nurses...the ones who have been here over a year, (can) take over the room so communication with them can sometimes be very hard."

The observations also revealed large variability and associated frustration on the part of nurses and physicians in the time it took for consultants to arrive. Consultants are typically physicians within the hospital whose expertise in areas such as orthopedics or neurosurgery is often critical to the management of patients with complex injuries. Both physicians and nurses attributed consultant delays to attitudes associated with particular specialties. In discussing this problem, one physician indicated "I always get frustrated by delays with consults. The consults are the weakest link... When I have a head injury I would like a neurosurgeon there before the head CT is done, but typically they want to wait until the head CT is done and I think we could all learn a lot more if we had the neurosurgeon present before that. They have one senior staffing the whole hospital and the VA, and they're understaffed." Nurses were often observed to be very upset over consult delays. As one nurse remarked, "I have seen nurses crying trying to get neurosurgery to come to see their patients; I think that is a big frustration."

The role of consultants is also an easily overlooked aspect of teamwork dynamics in the resuscitation setting. Discussions concerning the role of consultants illustrated how diffusion of these less localized medical providers into the resuscitation team can work against the precepts of teamwork and, in so doing, impact patient safety as well-an issue that was not apparent from our observations. As explained by one trauma attending, “The hardest cases are... when I need to get help from others and can't get it the way I want it when I want it. If it's a vascular process below the knee, if it's a hand problem, if it's any problem that I need a sub-specialist, I find those hard because you lose control of the trauma situation and you end up in a situation where people who don't understand trauma but only understand their sub-specialty will extend the case unnecessarily. So a urologist, for example, will try to fix a renal collection system in the same way that they would in a perfectly stable warm normal cancer patient whereas in a trauma patient things have to be done differently because you have to get the patient off the table and out of the room to stop the bleeding from ten other places...I find it hard when I have to rely on others." 
Another source of contention involving these "extended" (i.e., consultant) team members was the level of expertise that was being provided and the inherent delay in patient care that this exacted. As noted by this trauma attending, "Primarily the residents do it...but you have to accept that this is a teaching institution. You do not send your senior person most of the time because the junior person never gets any experience; then the junior person goes back and reports to the senior person, so there is a built-in delay in a teaching institution. That's part of training."

Another emergent theme from the observational study concerned the potential implications of organizational and administrative policies for patient care in the RU. In addition to the organization's ability to influence decisions regarding the availability and maintenance of equipment, its staffing policies were capable of adversely impacting teamwork dynamics. The unpredictability of patient arrivals results in enormous variability in the demand for medical providers, and the administration's current policies for addressing these demands by rotating nurses from other hospital units into resuscitation (rather than risk overstaffing during less busy times) often frustrated trauma physicians and nurses, as attested by the following comments of a trauma attending physician: "A cardiac surgery nurse does not know how to take care of a trauma case; half of what I do depends on the nurses and the scrub techs knowing what I am looking at so I don't have to be talking and delaying. I need the stuff now and they know what I am doing and they are hitting before I even ask for it. The nurses that come down here don't know where things are; if I need something they have to go search for it. That is unnecessary time to waste and sometimes critical time to waste...I know this is an effective business model and I am sure that is why they have done it but it...affects morale."

A trauma nurse also questioned the utility of the business model, especially in terms of its implications for patient safety: "They don't understand that if you have somebody not doing something for an hour or two right now (at least) you know then when we (get) all these people here you have enough people here to really give the proper care to the patient. When you call for help...they bring nurses from the ICU, so they are usually of the right skill level... but they can't really do acute resuscitation of patients, they really cannot do a room on their own because they don't have the training to do it, but they could help watch the patients in the observation area."

Overall, the resolution of these and other important issues within resuscitation will likely require interventions by, and collaborations among various stakeholders, including RU leaders, quality assurance managers, and hospital administrative officers. However, in acknowledging the need for such continuous improvement, we should not lose sight of the fact that these systems can be quite resilient, and in actuality there has been a steady reduction in the mortality rates at this trauma care system over the last five years. As one anesthesiologist commented after detailing some issues of concern, "...but let's be realistic...I think it (resuscitation) runs very well and we save a ton of lives."

\section{CONCLUSIONS}

Observation studies that are intended for revealing a broad array of potential issues as a basis for assessing the quality of patient management, and that target complex critical 
care settings that for various reasons are difficult for outside observers to penetrate and collect relevant data from, present methodological challenges. This article describes such an observation study that was performed within the resuscitation unit of a large level-1 trauma center. With careful attention to methodological details that include anticipating and preparing for logistical constraints, development of observation protocols, selection and training of observers, and employing a systematic approach for translating observations into data, it is believed that meaningful data can be derived from observations in these complex critical care environments. It is particularly in studies such as these that encompass broad goals related to the quality of patient management that the complementation of observation methods with interviews can produce a richer understanding of issues that, in turn, can serve as validation or reassessment of observational data and also as a basis for proposing recommendations for improving the quality of care.

We recognize that the findings from any individual level-1 trauma center may have varying degrees of relevance to other such facilities. Also, we acknowledge that having a limited number of observation periods may not have captured some important issues or considerations in patient care, and that various steps in the process of extracting data from observations are subject to individual variability. However, by highlighting the methodological challenges associated with collecting and examining observational data from this setting and the importance of complementing this data with other qualitative methods such as semi-structured interviews that are guided by findings from the observations, we believe that meaningful information can be provided both to health care workers in this area and to quality assurance programs that could be used to improve the delivery of care to patients with traumatic injuries.

\section{ACKNOWLEDGEMENT}

This work was supported by funding from the Department of Defense (Award Number W81XWH-05-1-0372). All opinions expressed in this paper are those of the authors and do not necessarily reflect the official opinion or position of the University of Miami or the Department of Defense. The authors thank Luis Labiste and Moravia Rodriguez for their contributions to the study and the staff at the Resuscitation Unit of the Ryder Trauma Center.

\section{REFERENCES}

1, National Trauma Institute, http://www.nationaltraumainstitute.com/home/trauma statistics.html, accessed December 10, 2009.

2. Agency for Healthcare Research and Quality, Costs of Treating Trauma Disorders Now Comparable to Medical Expenses for Heart Disease, AHRQ News and Numbers. Agency for Healthcare Research and Quality, Rockville, MD, January 25, 2006.

3. Xiao, Y., Moss, J., Mackenzie, C.F., Seagull, J.F. and Faraj, S., Transactive Responsibility Systems and High Reliability Teams: A Tentative Formulation, Proceedings of the Human Factors and Ergonomics Society $46^{\text {th }}$ Annual Meeting, 2002, 1428-1432.

4. Hoff, T.J. and Sutcliffe, K.M., Studying Patient Safety in Health Care Organizations: Accentuate the Positive. Joint Commission Journal on Quality and Patient Safety, 2006, 32, 5-15.

5. Sharit, J., A Modeling Framework for Exposing Risks in Complex Systems, Risk Analysis, 2000, 20(4), 469-482. 
6. Perrow, C., Normal Accidents: Living with High Risk Technologies. Princeton University Press, Princeton, NJ, 1999.

7. Hollnagel, E., Barriers and Accident Prevention, Ashgate, Aldershot, England, 2004.

8. Banister, B., Burman, E., Parker, I., Taylor, M. and Tindall, C., Qualitative Methods in Psychology: A Research Guide, Open University Press, Berkshire, England, 1994.

9. Willis, J.W., Foundations of Qualitative Research: Interpretive and Critical Approaches, Sage Publications, Thousand Oaks, CA, 2007.

10. Holloway, I., Qualitative Research in Health Care. Open University Press, Berkshire, England, 2005.

11. Lamond, D., The Information Content of the Nurse Change of Shift Report: A Comparative Study, Journal of Advanced Nursing, 2000, 31(4), 794-804.

12. Barker, K.N., Flynn, E.A., Pepper, G.A., Bates, D.W. and Mikeal, R.L, Medication Errors Observed in 36 Health Care Facilities, 2002, Archives of Internal Medicine, 162, 1897-1903.

13. Cook, R.I. and Woods, D.D., Adapting to New Technology in the Operating Room. Human Factors, 1996, 38(4), 593-611.

14. Cao, C.G.L. and Taylor, H., Effects of New Technology on the Operating Room Team, in H.M. Khalid, M.G. Helander and A.W.Yeo, eds., Work with Computing Systems, Damai Sciences, Kuala Lumpur, Malaysia, 2004, 309-312.

15. Fairbanks, R. J., Bisantz, A. M. and Sunm, M., Emergency department communication links and patterns. Annals of Emergency Medicine, 2007, 50(4), 396-406.

16. Carayon, P., Wetterneck, T. B., Hundt, A. S., Ozkaynak, M., DeSilvey, J., Ludwig, B., Ram, P. and Rough, S. S., Evaluation of nurse interaction with bar code medication administration technology in the work environment. Journal of Patient Safety, 2007, 3(1), 34-42.

17. Carthey, J., The Role of Structured Observational Research in Health Care, Quality and Safety in Health Care, 2003, 12 (supplement 2), ii13-ii16.

18. Nemeth, C. P., Cook, R. I. and Woods, D. D., The messy details: Insights from the study of technical work in healthcare. IEEE Transactions on Systems, Man, and Cybernetics - Part A: Systems and Humans, 2004, 34(6), 689-691.

19. The Joint Commission, Rights and Responsibilities of the Individual, http://www.jointcommission.org/AccreditationPrograms/Hospitals/Standards/09 FAQs/RI/Patient_Ri ghts and Informed.htm, accessed December 8, 2009.

20. Carayon, P. and Freisdorf, W., Human factors and Ergonomics in Medicine, in G. Salvendy, ed., Handbook of Human Factors and Ergonomics, third edn., John Wiley \& Sons, New York, 2006, 15171537.

21. Nemeth, C. P., Cook, R. I. and Wears, R. L., Studying the technical work of emergency care. Annals of Emergency Medicine, 2007, 50(4), 384-386.

22. Cicchettti, D. V. and Feinstein, A. R., High agreement but low Kappa: II resolving the paradoxes. Journal of Clinical Epidemiology, 1990, 43(6), 551-558.

23. Baker D. P., Salas, E., King, H., Battles, J., and Barach, P., The role of teamwork in the professional education of physicians: Current status and assessment recommendations. Joint Commission Journal on Quality and Patient Safety, 2005, 31(4), 185-202. 


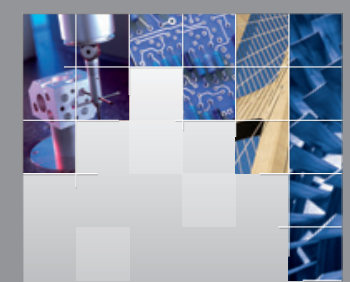

\section{Enfincering}
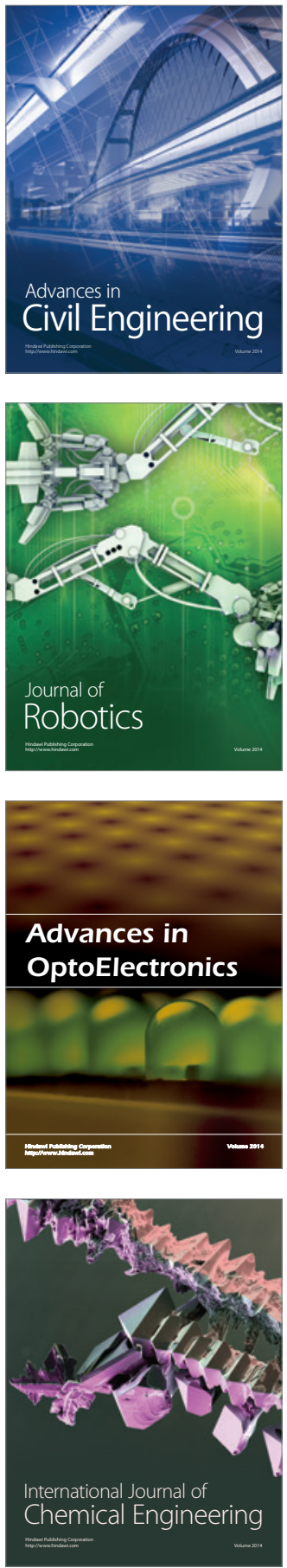

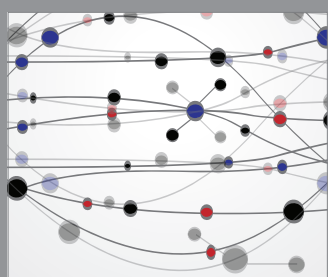

The Scientific World Journal

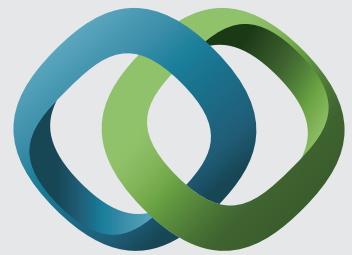

\section{Hindawi}

Submit your manuscripts at

http://www.hindawi.com
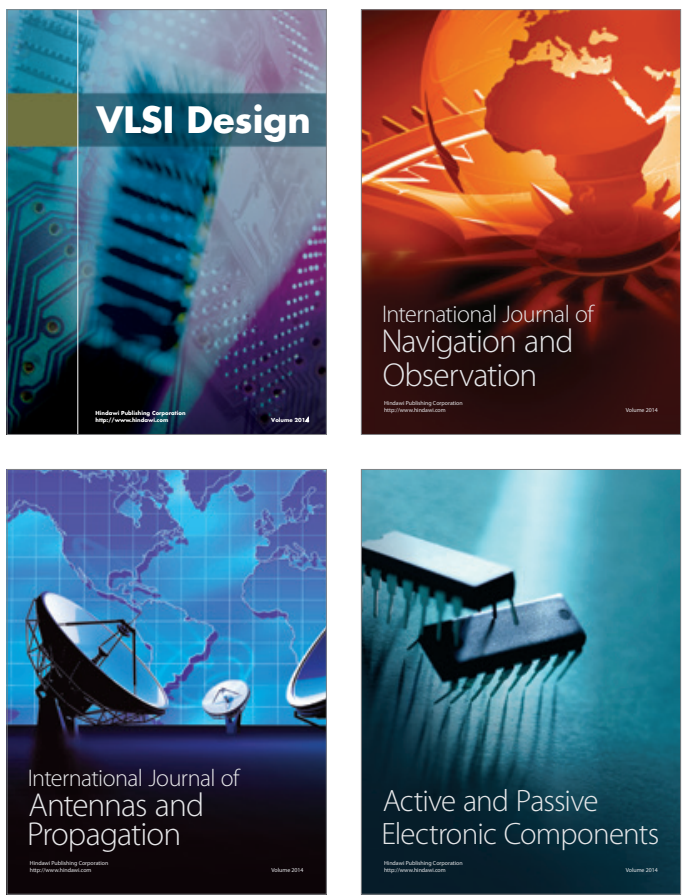
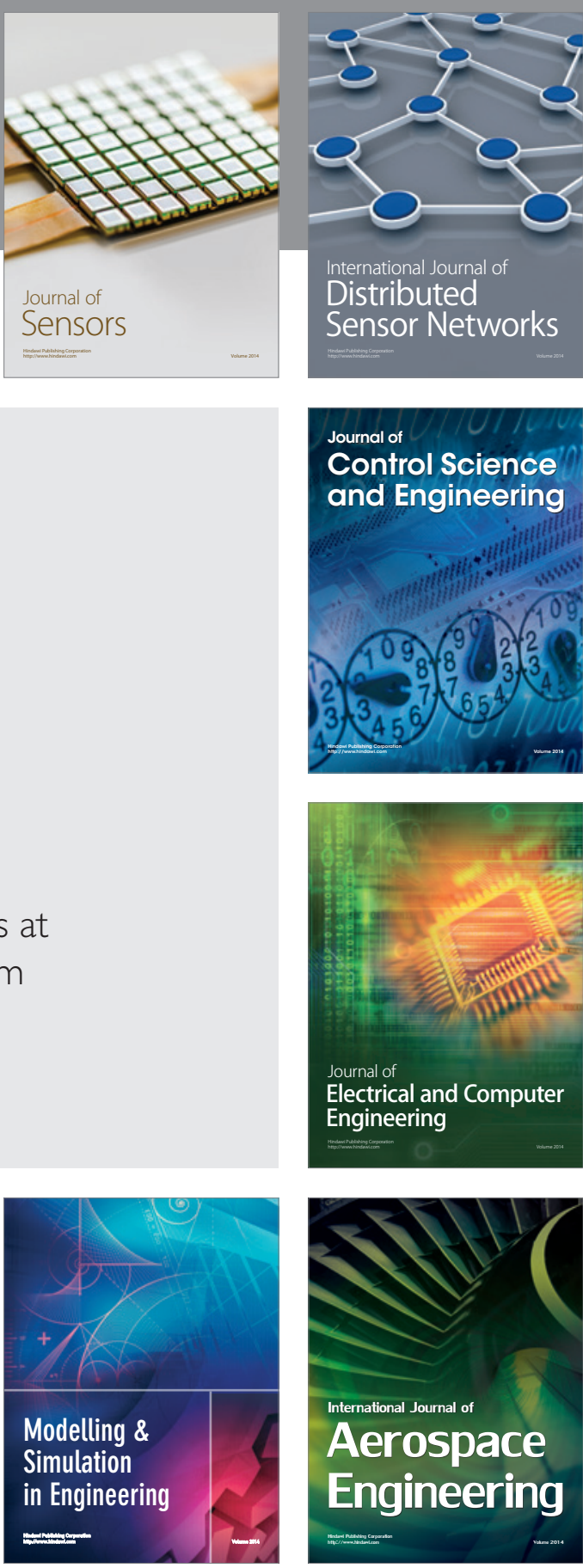

International Journal of

Distributed

Sensor Networks

Journal of

Control Science

and Engineering
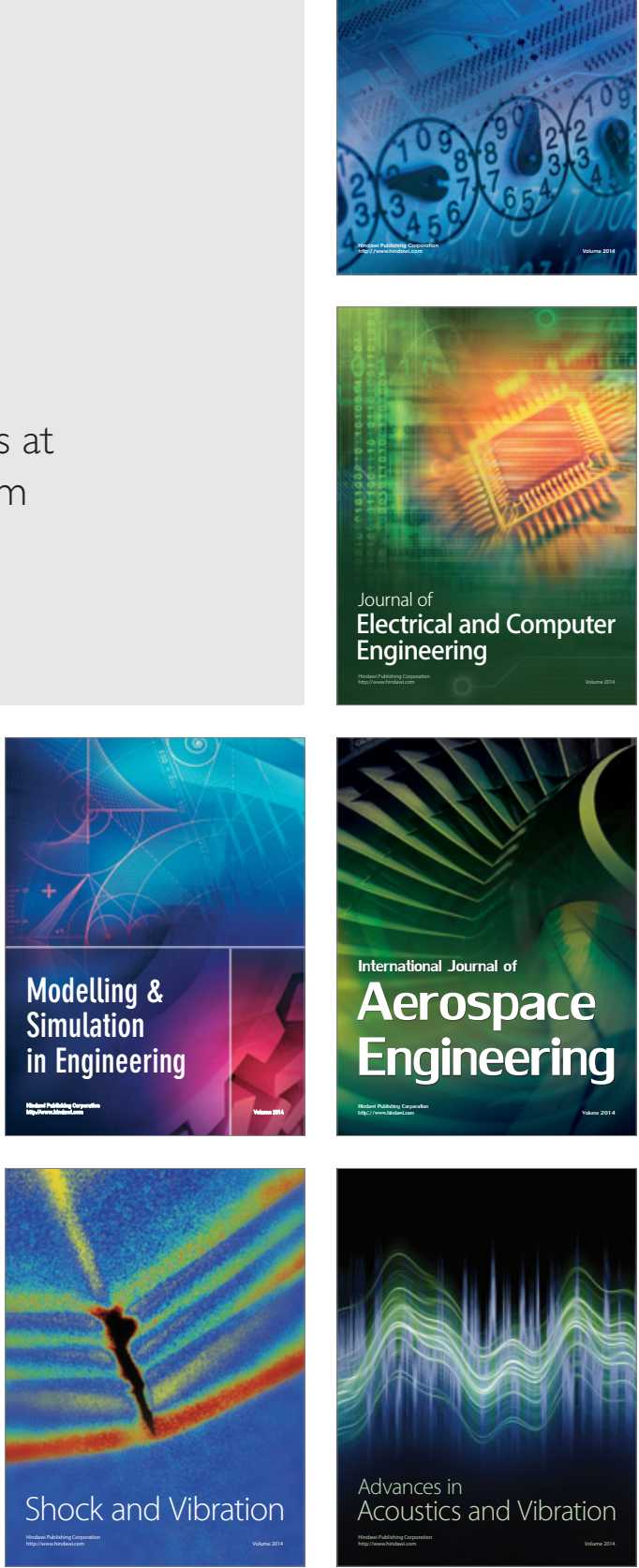